\title{
Efficient exon skipping of SCCG mutations mediated by phosphorodiamidate morpholino oligomers
}

\author{
Eugene J. Wyatt, ${ }^{1}$ Alexis R. Demonbreun, ${ }^{1}$ Ellis Y. Kim, ${ }^{2}$ Megan J. Puckelwartz, ${ }^{1}$ Andy H. Vo, ${ }^{3}$ \\ Lisa M. Dellefave-Castillo, ${ }^{1}$ Quan Q. Gao, ${ }^{4}$ Mariz Vainzof, ${ }^{5}$ Rita C. M. Pavanello, ${ }^{5}$ Mayana Zatz, ${ }^{5}$ \\ and Elizabeth M. McNally' \\ ${ }^{1}$ Center for Genetic Medicine, Northwestern University Feinberg School of Medicine, Chicago, Illinois, USA. ${ }^{2}$ Committee on \\ Molecular Medicine and Molecular Pathogenesis and ${ }^{3}$ Committee on Developmental Biology and Regenerative Medicine, \\ The University of Chicago, Chicago, Illinois, USA. ${ }^{4}$ Division of Hematology/Oncology, Northwestern University Feinberg \\ School of Medicine, Chicago, Illinois, USA. ${ }^{5}$ Human Genome and Stem-Cell Center, Institute of Biosciences, University of \\ São Paulo, São Paulo, Brazil.
}

Exon skipping uses chemically modified antisense oligonucleotides to modulate RNA splicing. Therapeutically, exon skipping can bypass mutations and restore reading frame disruption by generating internally truncated, functional proteins to rescue the loss of native gene expression. Limb-girdle muscular dystrophy type $2 \mathrm{C}$ is caused by autosomal recessive mutations in the SCCC gene, which encodes the dystrophin-associated protein $\gamma$-sarcoglycan. The most common SCCC mutations disrupt the transcript reading frame abrogating $\gamma$-sarcoglycan protein expression. In order to treat most SCCG gene mutations, it is necessary to skip 4 exons in order to restore the SCCG transcript reading frame, creating an internally truncated protein referred to as MiniGamma. Using direct reprogramming of human cells with MyoD, myogenic cells were tested with 2 antisense oligonucleotide chemistries, 2'-0-methyl phosphorothioate oligonucleotides and vivo-phosphorodiamidate morpholino oligomers, to induce exon skipping. Treatment with vivo-phosphorodiamidate morpholino oligomers demonstrated efficient skipping of the targeted exons and corrected the mutant reading frame, resulting in the expression of a functional MiniGamma protein. Antisense-induced exon skipping of SCCG occurred in normal cells and those with multiple distinct SCCC mutations, including the most common $521 \Delta \mathrm{T}$ mutation. These findings demonstrate a multiexon-skipping strategy applicable to the majority of limb-girdle muscular dystrophy $2 \mathrm{C}$ patients.

Conflict of interest: EMM and EJW are coinventors of a patent related to exon skipping (US patent 9,777,271).

Submitted: December 19, 2017 Accepted: April 5, 2018 Published: May 3, 2018

Reference information: JCI Insight. 2018;3(9):e99357. https:// doi.org/10.1172/jii.insight.99357.

\section{Introduction}

Limb-girdle muscular dystrophy type 2C (LGMD 2C) is a debilitating muscle disease characterized by progressive muscle degeneration, loss of ambulation, and eventual deterioration of respiratory and cardiac function $(1,2)$. Autosomal recessive mutations in the $\gamma$-sarcoglycan gene (SGCG) cause LGMD 2C (3). The SGCG gene encodes a type II transmembrane protein that is a component of the sarcoglycan complex, which - in skeletal and cardiac muscle - is comprised of $\alpha-, \beta-, \gamma-$, and $\delta$-sarcoglycans $(4,5)$. The sarcoglycan complex is part of the larger dystrophin glycoprotein complex (DGC), which localizes to the muscle plasma membrane, stabilizes the sarcolemma, and connects the extracellular matrix to the intracellular cytoskeleton $(6,7)$. SGCG mutations commonly disrupt the reading frame and result in the absence of $\gamma$-sarcoglycan protein expression $(3,8,9)$. There are currently no approved therapies for treating LGMD $2 \mathrm{C}$, and the mainstay of medical treatment relies on supportive care (10).

Exon skipping uses chemically modified antisense oligonucleotides (AONs). The utility of exon skipping to correct reading frame disruption has been demonstrated for the treatment of Duchenne muscular dystrophy (DMD), which is caused by mutations in the dystrophin gene (11). The concept of exon skipping derived from observations that deletions in the dystrophin gene, which disrupt the transcript reading frame, cause the severe DMD, while in-frame deletions cause Becker muscular dystrophy (BMD), a milder form of disease with improved clinical outcomes $(12,13)$. Moreover, sporadic dystrophin-positive fibers, known 
as revertant fibers, have been observed in muscle biopsies from DMD patients and in mouse models of the disease $(14,15)$. Revertant fibers in dystrophin mutant muscle represent intrinsic bypass of mutations that correct the reading frame to restore expression of an internally deleted dystrophin protein (16). Exon skipping uses AONs to hinder sterically the pre-mRNA splicing of a target exon, blocking its incorporation into the mature mRNA transcript (17). For clinical benefit, the mRNAs produced after exon skipping are required to encode and produce an internally truncated protein with functional relevance.

AONs are short RNA or DNA oligomers that are the reverse complementary sequence to a target mRNA sequence (18). AON chemical modifications are designed to avoid nuclease degradation and enhance RNA binding (18). Further modifications are required for the purpose of splice modulation to avoid RNase-H degradation of the oligo-RNA hybrid, as the goal is transcript correction and not destruction (17). Multiple chemical modifications were developed for this purpose, with 2'-O-methyl phosphorothioate (2OMePS) AONs and phosphorodiamidate morpholino oligomers (PMOs) having been tested in human clinical trials for DMD (19-22). In 2016, the US Food and Drug Administration (FDA) granted accelerated approval of a PMO to treat DMD caused by specific mutations in the dystrophin gene, whose reading frame is corrected through the skipping of exon 51 (23). Eteplirsen represented an achievement in the fields of neuromuscular disease and molecular gene correction, and it was followed by the approval of nusinersen for treating spinal muscular atrophy (SMA) $(23,24)$. Progress toward clinical development of antisense-mediated splice modulating therapy has expanded beyond the treatment of DMD to include other disorders, such as Pompe disease, cystic fibrosis, cardiomyopathies, and laminopathies (25-29).

Recently, we explored the therapeutic potential of exon skipping to treat LGMD $2 \mathrm{C}$ by examining whether Mini-Gamma protein can successfully assume the functional roles of full-length $\gamma$-sarcoglycan (30). The $S G C G$ gene is composed of 8 exons, with exons $2-8$ encoding protein. The most common $S G C G$ mutation is a single thymine deletion from a string of 5 thymines in $S G C G$ exon 6 , designated $521 \Delta \mathrm{T}$ (3, 8). Reframing the $521 \Delta \mathrm{T}$ transcript requires the skipping of exons $4,5,6$, and 7 , creating an internally truncated product encoded by exons 2, 3, and 8, termed Mini-Gamma. We demonstrated that transgenic expression of murine Mini-Gamma provided functional and pathological benefit to correct the loss of $\gamma$-sarcoglycan in both fly and mouse models of the disease (30). Here, we report the use of AON-mediated exon skipping to correct the reading frame of multiple SGCG mutations, including the $521 \Delta \mathrm{T}$ mutation, in a human cell model of disease. To this end, multiexon-skipping AON cocktails were employed using either $20 \mathrm{MePS}$ or vivo-PMO chemistries. The vivo-PMOs were more efficient than $20 \mathrm{MePS}$ oligomers in generating the Mini-Gamma transcript. This approach targets any mutation occurring in SGCG exons 4-7, making this therapy applicable to the majority of LGMD 2C patients.

\section{Results}

An exon-skipping strategy to treat LGMD 2C. The $8 \gamma$-sarcoglycan exons produce a type II transmembrane protein that is an essential component of the muscle plasma membrane. Homozygous $S G C G$ mutations, which disrupt the reading frame, generate premature stop codons in the mRNA, preventing protein expression and causing LGMD 2C (Figure 1A). Exon skipping uses AONs to block pre-mRNA splicing sites, bypassing mutations and modifying the exon content of an mRNA. Maintaining the reading frame requires skipping of exons 4, 5, 6 , and 7 to create an internally truncated transmembrane protein, termed Mini-Gamma, encoded by exons 2, 3, and 8 (Figure 1B). Mini-Gamma retains the essential intracellular, transmembrane, and extracellular SGCG domains required for functionality (Figure 1C), and transgenic expression of murine Mini-Gamma has been shown to rescue multiple features of muscular dystrophy in fly and mouse models of disease (30).

Chemical modifications of AONs are necessary to prevent nuclease attack and degradation of the transcript, while increasing their stability and interaction with the RNA target $(17,18)$. We evaluated 2 common AON chemical modifications for exon skipping for LGMD 2C: 2OMePS and PMO (Figure 1D). PMOs were synthesized as vivo-PMOs, which contained a covalently linked C-terminal octa-guanidine dendrimer delivery moiety that promotes cell membrane permeability (31). Overall, this exon-skipping strategy was designed to target SGCG missense, nonsense, or frame-shifting mutations in exons 4-7 that account for the majority of LGMD $2 \mathrm{C}$ disease-causing mutations.

$A$ human cell-based model of LGMD 2C. Fibroblasts were obtained from an individual with an autosomal recessive deletion of a single thymine in $S G C G$ exon 6 , a mutation designated $521 \Delta \mathrm{T}$, which disrupts the reading frame and results in the absence of $\gamma$-sarcoglycan protein (Figure 2A) (3). This is the most common mutation of the $S G C G$ gene and accounts for almost half of all LGMD 2C patients $(3,8)$. We reprogrammed con- 
A
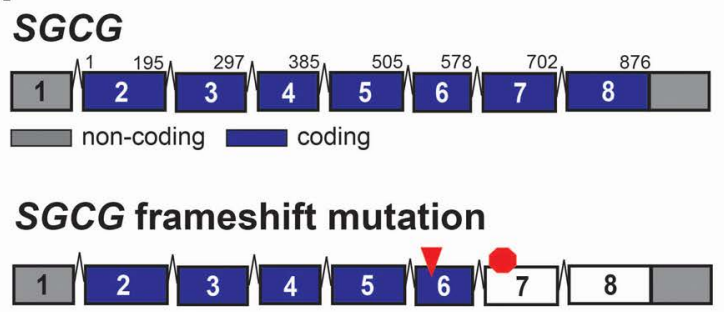

$\nabla$ frameshift premature stop

C

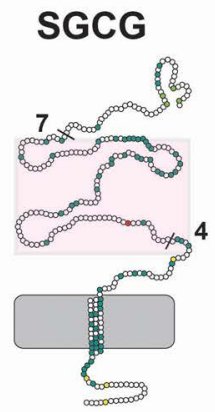

\section{Mini-Gamma}

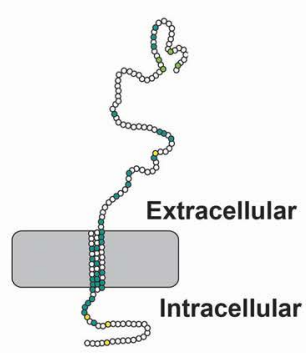

B

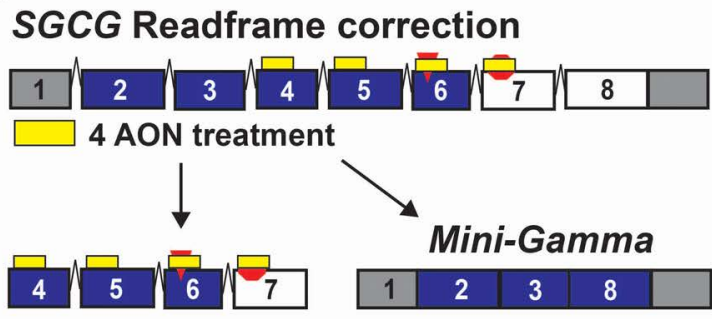

Figure 1. An exon-skipping strategy to treat LGMD 2C. (A) The SGCG gene, which encodes $\gamma$-sarcoglycan, is comprised of 8 exons, and many SGCG mutations disrupt the transcript reading frame, causing Limb-girdle muscular dystrophy (LCMD) 2C. (B) Correction of the reading frame requires the skipping of SGCG exons 4, 5, 6, and 7 at the pre-mRNA level. Four antisense oligonucleotides (AON) were designed to exclude these exons from the mature mRNA transcript. The bypass of exons 4-7 generates an internally truncated product termed Mini-Gamma encoded by exons 2, 3, and 8. (C) Mini-Gamma protein lacks a portion of the extracellular domain encoded by exons 4-7 (red box) and retains the essential cytosolic, transmembrane, and extracellular domains required for functionality (30). (D) Exon skipping AONs are chemically modified to avoid nuclease degradation and destruction of the mRNA transcript. Shown are the chemical structures of 2 types of AONs used for exon skipping. 2'-0-methyl phosphorothioates (2OMePS) include a methyl group on the ribose ring and substitution of sulfur for oxygen to create a phosphothioate bond (red). Phosphorodiamidate morpholino oligomers (PMOs) incorporate morpholino rings rather than ribose rings and substitute nitrogen for oxygen to create a phosphorodiamidate bond (blue) (18). Vivo-PMOs are synthesized with a covalently linked 3' octa-guanidine delivery moiety for enhanced cell delivery (31).

trol and $521 \Delta \mathrm{T}$ fibroblasts into the myogenic lineage using a tamoxifen-inducible MyoD (iMyoD) lentiviral system (32). To assess the possibility of reading frame correction via exon skipping, we first determined if the reprogrammed $521 \Delta \mathrm{T}$ cells produce a stable mutant mRNA transcript. Analysis of PCR-amplified transcripts detected native and mutant $S G C G$ expression in reprogrammed control and $521 \Delta \mathrm{T}$ fibroblasts, respectively (Figure 2B) (See complete unedited blots in the supplemental material; supplemental material available online with this article; https://doi.org/10.1172/jci.insight.99357DS1). Sequence analysis confirmed the 521 $\Delta \mathrm{T}$ deletion in the mutant transcript (Supplemental Figure 1, A and B). Each cell line was efficiently induced into the myogenic lineage, as demonstrated by the expression of desmin and the presence of elongated myotube-like structures (Figure 2C). The expression of the $\gamma$-sarcoglycan protein was observed in reprogrammed control cells and absent in the $521 \Delta \mathrm{T}$ patient cells, commensurate with mutation status (Figure 2D).

$2 O M e P S$ AON exon skipping of the $521 \Delta T$ transcript. The $20 \mathrm{MePS}$ AON chemical modification is among the most commonly used for exon-skipping splice manipulation and has efficiently corrected dystrophin frameshift mutations in cell models of DMD (33-35). For this LGMD 2C strategy, 2OMePS AONs targeting intraexonic regions in SGCG exons 4, 5, 6, and 7 (Table 1) were designed in accordance with previously described guidelines (36). Transfection of reprogrammed $521 \Delta \mathrm{T}$ cells with individual $2 O \mathrm{MePS}$ AONs demonstrated skipping of individual exons 5 and 7, partial skipping of exon 4, and indeterminate skipping of exon 6 (Supplemental Figure 2, A and B). We also evaluated whether the normal $S G C G$ sequence was targeted by AON-mediated exon skipping. Transfection of reprogrammed control cells with single $2 O \mathrm{MePS}$ elicited a similar skipping profile as 521 $\Delta \mathrm{T}$ SGCG cells (Supplemental Figure $2 \mathrm{C}$ ), indicating that mutation in $S G C G$ is not required for exon skipping.

Cells were then treated with a 4-AON exon-skipping cocktail aimed at $S G C G$ exons 4, 5, 6, and 7 (Supplemental Table 1). Analysis of PCR-amplified transcripts after 36 hours of treatment showed the expression 
A
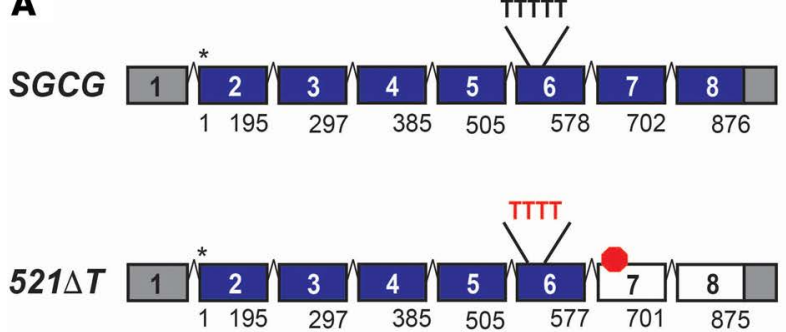

B

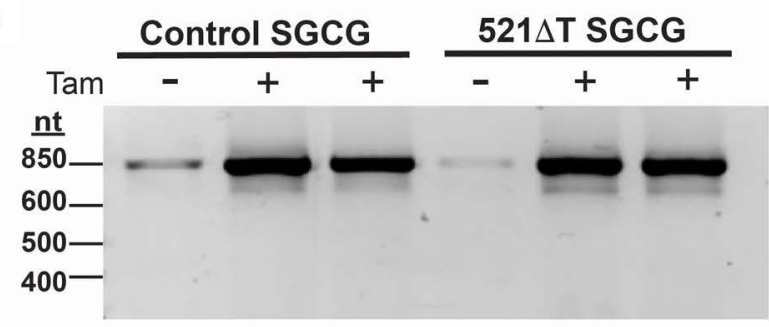

C

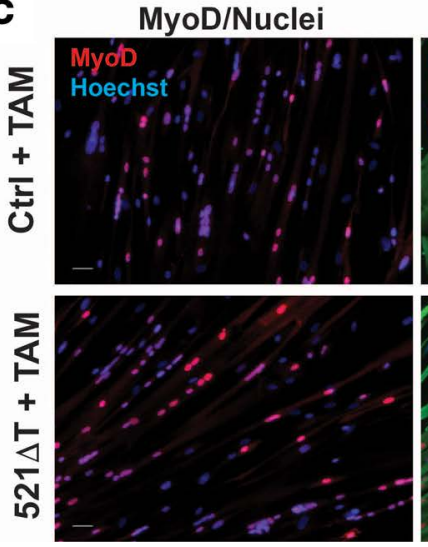

Desmin
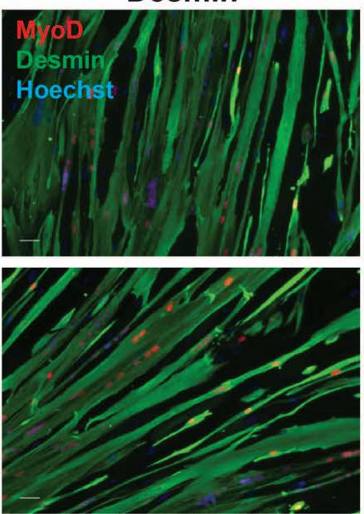

D

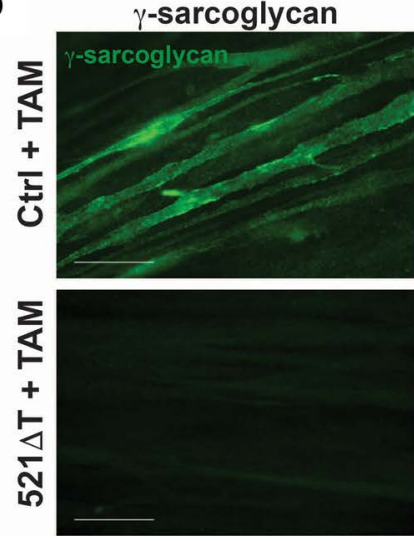

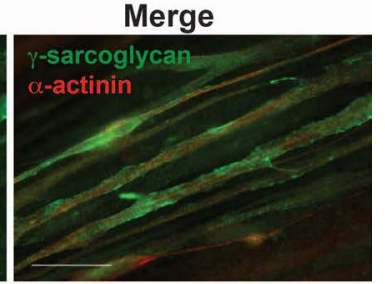

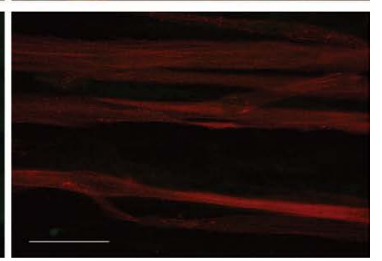

Figure 2. SGCG mRNA and protein expression in fibroblasts reprogrammed into the myogenic lineage. (A) Schematic of the SCCG pre-mRNA transcript. The most common LGMD 2C mutation is a single thymine (T) deletion from a string of 5Ts in SCCG exon 6. This mutation, designated 521 $\triangle \mathrm{T}$, results in a transcript frame shift, which generates a premature stop codon (red octagon). Asterisks represent the transcription start sites, and the numbers denote the mRNA region encoded by each exon. Fibroblasts from a normal control subject (SGCG intact) and a LCMD 2C individual with the 521 $\triangle T$ mutation were directly reprogrammed into the myogenic lineage using a tamoxifen (Tam) inducible MyoD lentiviral construct (iMyoD) (32). (B) RT-PCR analysis demonstrated expression of the SCCG mRNA in normal control and 521 $\triangle$ T cells after tamoxifen induction and culture in differentiation media ( $5 \mu \mathrm{M} 40 \mathrm{H}$-tamoxifen, 48 hours, 9 days differentiation). (C) Immunofluorescence microscopy (IFM) demonstrated the efficient transduction and myogenic reprogramming of both the control and $521 \Delta \mathrm{T}$ cell lines ( $5 \mu \mathrm{M} 40 \mathrm{H}$-tamoxifen, 48 hours, 12 days differentiation). Nuclear expression of MyoD (red) is observed in the iMyoD-transduced cells. Reprogrammed cells expressed the myogenic marker desmin (green) and formed multinucleated myotubes. Nuclei were labeled with Hoechst 3342 (blue). Scale bar: $50 \mu \mathrm{M}$. (D) $\gamma$-Sarcoglycan protein (green) was readily detected in the reprogrammed control cells, but not in reprogrammed $521 \Delta T$ cells, in accordance with their mutation status. Expression of $\alpha$-actinin (red) indicated the myogenic reprogramming of each cell line. Scale bar: $50 \mu M$.

of multiple intermediate products, which represented the skipping of 1, 2, and 3 exons (Figure 3A). Notably, there was minimal expression of the 4-exon-skipped Mini-Gamma transcript at the expected size (Figure 3A). Similar treatment of control cells produced intermediate transcripts with no evidence of Mini-Gamma encoding mRNA expression after 48 hours (Figure 3B). Collectively, these data indicate that reading frame correction of the $521 \Delta \mathrm{T} S G C G$ frameshift mutation with the $20 \mathrm{MePS}$ AON multiexon-skipping strategy was only modestly effective due to inefficient skipping of individual SGCG exons, specifically exon 6.

Vivo-PMO exon skipping of the 5214T transcript. PMOs are another commonly used AON chemistry used for exon skipping. These molecules carry no net charge and appear nontoxic with minimal off-target interactions (37). In order to improve cellular uptake in vitro, PMOs were covalently conjugated to an octa-guanidine dendrimer used to generate vivo-PMOs. AONs using vivo-PMO chemistry were designed as described and targeted intraexonic regions in $S G C G$ exons 4, 5, 6, and 7 (Table 1). Transfection of reprogrammed $521 \Delta \mathrm{T}$ cells with single vivo-PMOs demonstrated dose-dependent skipping of individual SGCG exons 4, 5, 6, and 7 (Supplemental Figure 3A). Transfection of control cells with single vivo-PMOs demonstrated similar dose-dependent skipping of individual $S G C G$ exons 4, 5, 6, and 7, which indicated the ability of vivo-PMOs to efficiently skip exons of the native SGCG transcript (Supplemental Figure $3 \mathrm{~B}$ ).

Additionally, there was evidence of double exon skipping after treatment with single vivo-PMOs targeting exons 4, 5, and 6 (Supplemental Figure 3, A and B), likely due to the dual skipping of individual exons 4,5 , and 6 coupled with the endogenous skipping of exon 7. Notably, single vivo-PMO treatments targeting 
Table 1. SGCG exon targets and AON sequences

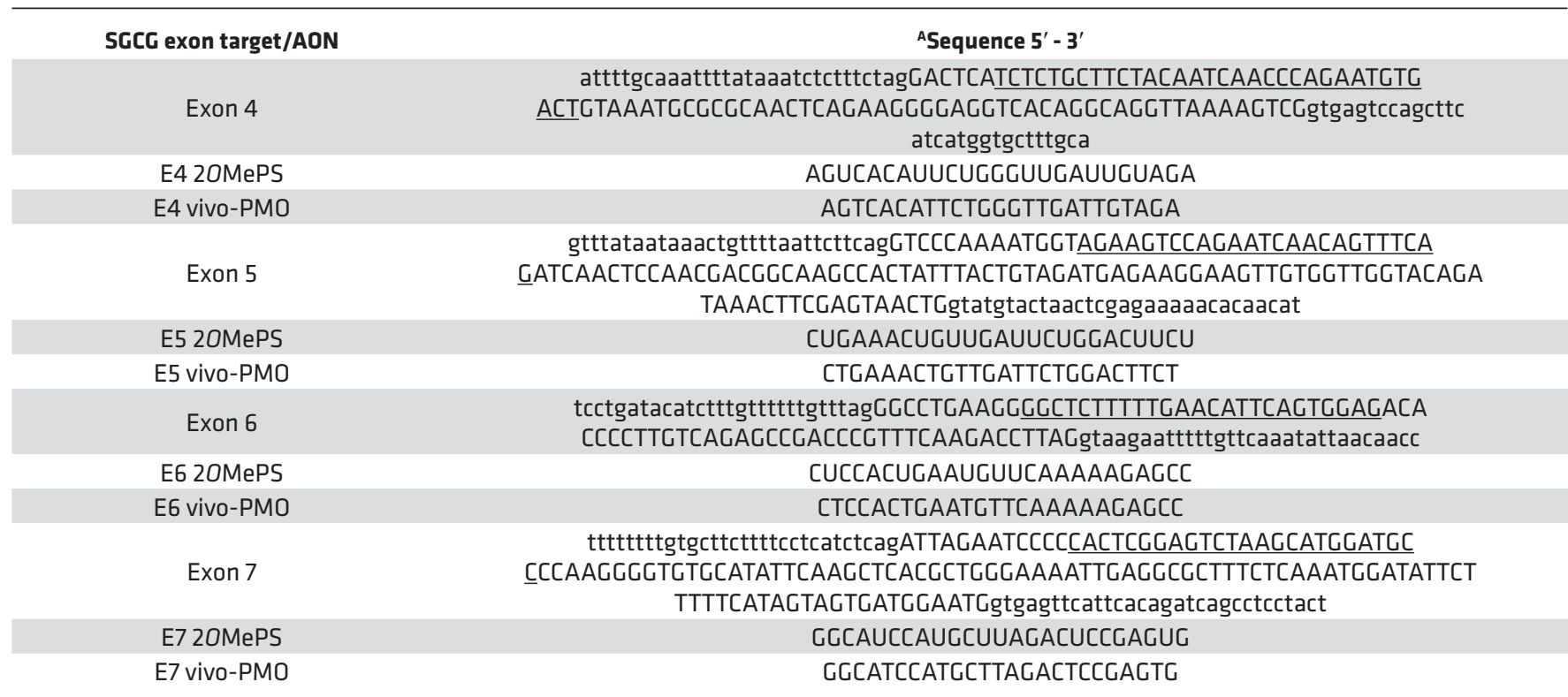

Andividual exons are shown as upper case, with 30 bp of flanking intronic sequence shown as lower case. AON target sequences are underlined.

exon 7 did not demonstrate double skipping (Supplemental Figure 3, A and B). Splice-site analysis of $S G C G$ exon 7 revealed 2 potential regions of splice repression that were conserved between the human and mouse SGCG sequences (Supplemental Figure 3C).

To correct the SGCG $521 \Delta \mathrm{T}$ reading frame disruption and generate a Mini-Gamma transcript, reprogrammed cells were treated with a 4-AON cocktail of vivo-PMOs targeting $S G C G$ exons 4, 5, 6, and 7 (Supplemental Table 1). Analysis of PCR-amplified transcripts after 36-hour treatment showed predominant expression of the reading frame-corrected, 4-exon-skipped Mini-Gamma transcript with minimal expression of intermediate products (Figure 4A). Treatment of control myogenic cells with the same vivo-PMO cocktail also elicited predominant expression of the Mini-Gamma transcript (Figure 4B). The internally truncated Mini-Gamma products were confirmed by sequence analysis (Figure 4, C and D). These results demonstrated efficient reading frame correction of the SGCG 521 $\triangle \mathrm{T}$ mutation with our multiexon-skipping strategy and the ability of vivo-PMOs to skip the native $S G C G$ transcript. Furthermore, the data indicated improved efficiency of exon skipping with vivo-PMOs compared with $20 \mathrm{MePS}$ AONs in the cell model.

Reading frame correction of multiple SGCG frameshift mutations. We next examined the ability of this exon-skipping strategy to correct unique SGCG frameshift mutations. Fibroblasts were obtained from 4 different LGMD 2C patients harboring $3 S G C G$ frameshift mutations. The first was from an individual with a large deletion of $S G C G$ encompassing the entire $S G C G$ gene and a second allele missing only exons 5 and 6 (ex5/6del). Two other cell lines were from 2 siblings with a large deletion of the entire SGCG gene on one allele and a deletion of only exon 6 (ex6del) on the second allele; the fourth was an individual with a homozygous deletion of $S G C G$ exon 7 (ex7del) (Figure 5, A-C). Each cell line was reprogrammed into the myogenic lineage using the $\mathrm{iMyoD}$ system. To generate the reading frame-corrected Mini-Gamma transcripts for each mutation, the individual LGMD 2C myogenic cell cultures were treated with a mutation-specific cocktail of vivo-PMOs (Figure 5, A-C, and Supplemental Table 1). This mutation-specific approach limited the total AON exposure to that required for reading frame correction. Analysis of PCR-amplified transcripts after 36-hour treatment showed predominant expression of the internally truncated reading frame-corrected Mini-Gamma transcript in each of the LGMD 2C patient cell lines (Figure $5, \mathrm{~A}-\mathrm{C})$. Sequencing analysis confirmed the patient-specific mutations and the generation of Mini-Gamma after vivo-PMO treatment (Figure 5, D-F). These findings demonstrate the ability to correct multiple $S G C G$ frameshift mutations with this exon-skipping strategy.

Restoration of Mini-Gamma protein expression was demonstrated in the 521 $\Delta \mathrm{T}$, ex5/6del, and ex6del mutations. Immunofluorescence microscopy (IFM) detected $\gamma$-sarcoglycan protein in reprogrammed 
A

2OMePS exon skipping of the $521 \Delta \mathrm{T}$ transcript
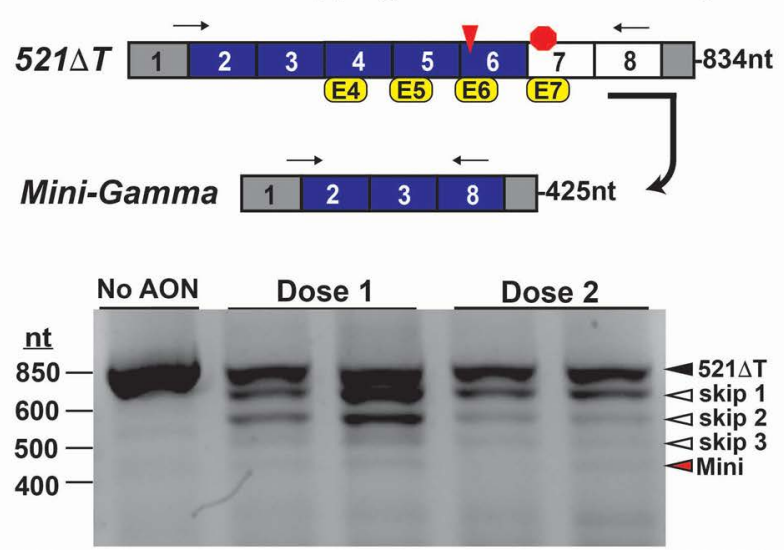

B

2OMePS exon skipping of the SGCG transcript
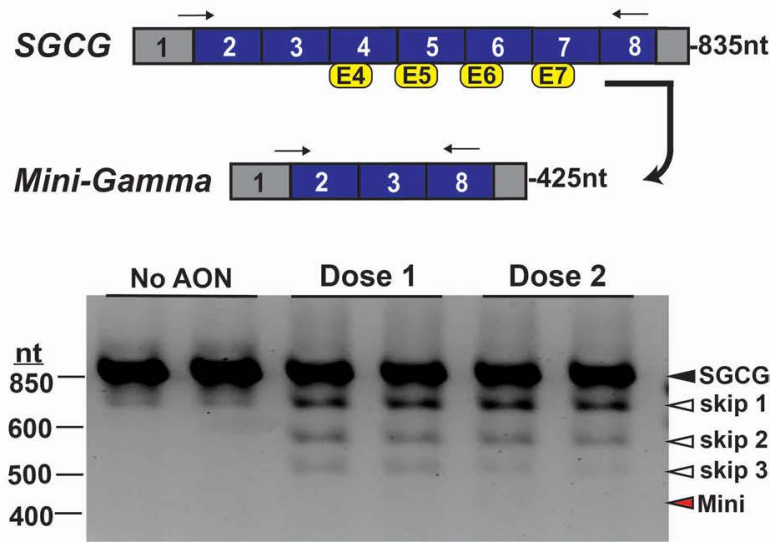

Figure 3. 20MePS-mediated exon skipping of the SCCG transcript. (A) Correction of the SCCG 521 $\triangle$ T frameshift mutation (red triangle) requires a multiAON exon-skipping strategy targeting exons 4, 5, 6, and 7 (yellow boxes) to generate the reading frame-corrected Mini-Gamma transcript encoded by exons 2, 3, and 8. Arrows denote the location of the RT-PCR primers, and the expected amplicon lengths are indicated. Myogenic cells were exposed to a multiexon-skipping cocktail of 2OMePS AONs targeting exons 4, 5, 6, and 7. RT-PCR analysis demonstrated the expression of the $521 \Delta \mathrm{T}$ transcript (black arrowhead), along with PCR products representing the skipping of 1,2 , and 3 exons (white arrowheads). A PCR product representing the 4-AON-mediated generation of the Mini-Gamma was observed at the expected size (red arrowhead). (B) Treatment of reprogrammed normal control fibroblasts with $20 \mathrm{MePS}$ AONs targeting exons 4, 5, 6, and 7 also induced exon skipping. RT-PCR analysis demonstrated the expression of the SCCG transcript (black arrowhead), along with PCR products representing the skipping of 1, 2, and 3 exons (white arrowheads). No PCR product representing the 4-AON-mediated generation of the Mini-Gamma was observed at the expected size (red arrowhead).

LGMD 2C patient myotubes 3 days after treatment with reading frame-correcting vivo-PMOs (Figure $6, \mathrm{~A}, \mathrm{C}$, and E). Similarly conducted and acquired imaging did not detect $\gamma$-sarcoglycan protein expression in reprogrammed patient cells treated with a nontargeting control vivo-PMO. Quantitative image analysis confirmed the expression of Mini-Gamma in cells transfected with reading frame-correcting vivo-PMO cocktails as compared with cells treated with nontargeting control vivo-PMOs (Figure 6, B, D, and F). These findings demonstrate the ability to correct multiple SGCG frameshift mutations with this exon-skipping strategy, restoring Mini-Gamma expression at the protein level. Detection of Mini-Gamma protein by immunoblot was limited by the culture system, as reprogrammed control fibroblasts expressed only modest levels of even normal $\gamma$-sarcoglycan protein (Supplemental Figure 4A). Mini-Gamma protein expression was nonuniform across the culture system, with some myotubes expressing more Mini-Gamma protein (Figure 6, A-F). The ex5/6del line had the highest levels of Mini-Gamma expression, as seen by IFM (Figure 6, C and D). Correspondingly, Mini-Gamma was detected via immunoblot in reprogrammed ex5/6del cells after reading frame correction with vivo-PMOs (Supplemental Figure 4B).

The sarcoglycan complex includes $\alpha-, \beta-, \delta$-, and $\gamma$-sarcoglycans, with $\beta$-, $\delta$-, and $\gamma$-sarcoglycans forming the core unit $(4,5)$. The absence of $\gamma$-sarcoglycan reduces the expression of $\beta$ - and $\delta$-sarcoglycan at the sarcolemma in intact muscle (21). To determine if Mini-Gamma stabilized the sarcoglycan complex, $\beta$ - and $\delta$-sarcoglycan expression levels were examined after treatment with reading frame-correcting vivoPMOs. An increase of $\beta$ - and $\delta$-sarcoglycan protein expression was detected by IFM in those myotubes that expressed Mini-Gamma protein (Supplemental Figure 5, A-C, and Supplemental Figure 6, A-C).

Functional reading frame correction in SGCG reprogrammed cells. Previously, we showed the ability to directly reprogram urine-derived cells (UDC) into the myogenic lineage through the overexpression of MyoD (38). To further analyze this SGCG exon-skipping strategy, we obtained UDCs from an LGMD $2 \mathrm{C}$ patient harboring a frameshift mutation caused by the deletion of SGCG exon 6 (ex6del). Normal control and ex6del UDCs were reprogrammed into the myogenic lineage, seen as $\alpha$-actinin expression and the appearance of elongated myotube-like structures (Figure 7A). $\gamma$-Sarcoglycan protein expression was detected in the reprogrammed normal control cells but not in LGMD 2C cells, commensurate with SGCG mutation status (Figure 7A). To generate the reading frame-corrected Mini-Gamma transcript, ex6del myogenic cells were treated with vivo-PMOs targeting exon 4, 5, and 7 (Supplemental Table 1). Analysis of PCR-amplified transcripts after 24 and 48 hours showed predominant expression of the 
A

Readframe correction of SGCG $521 \Delta T$
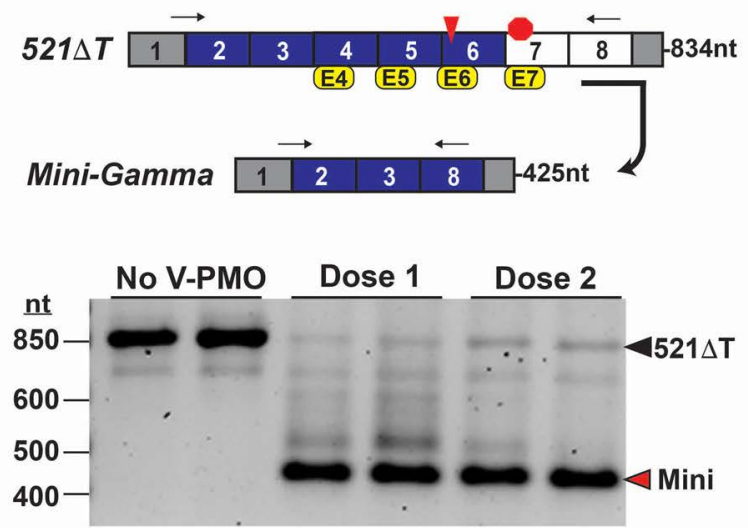

C

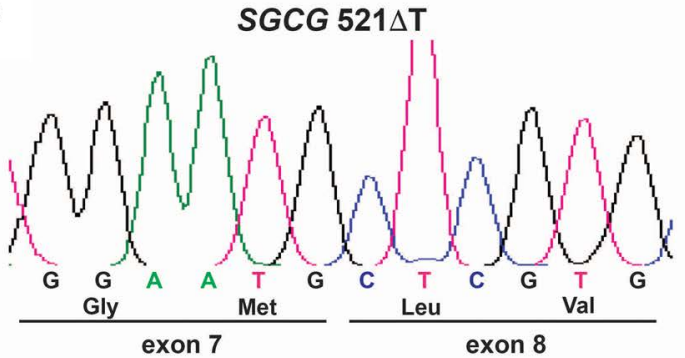

$521 \Delta T$ Mini-Gamma

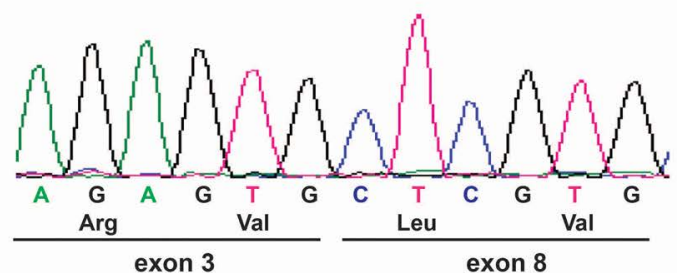

Exon skipping of the SGCG transcript
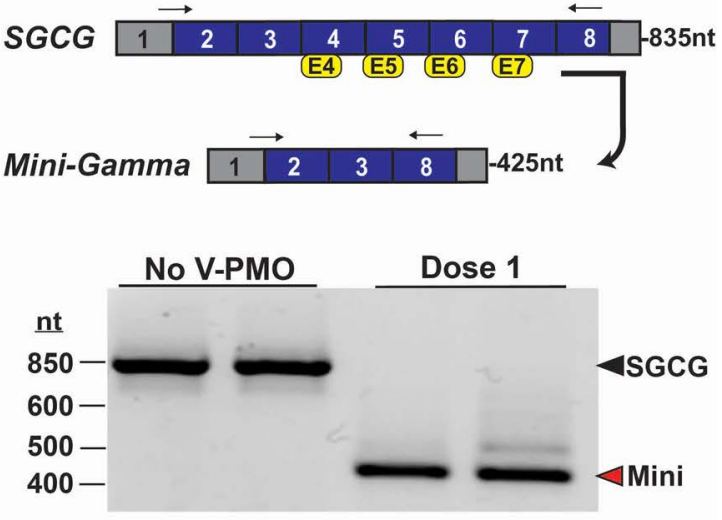

D

SGCG Control

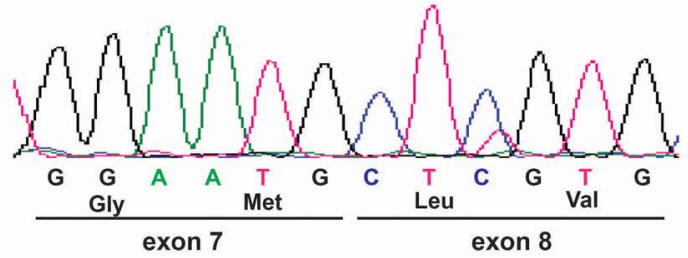

Control Mini-Gamma

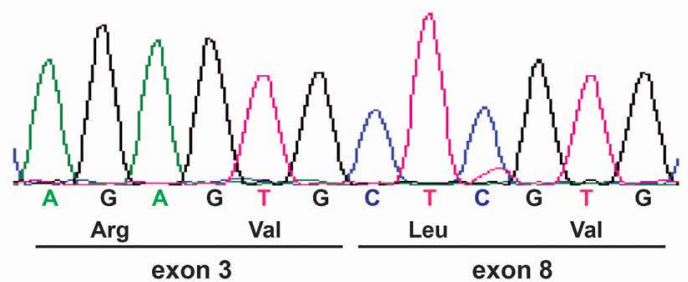

Figure 4. Vivo-PMO-mediated exon skipping of the SGCG transcript. (A) A multi-AON exon-skipping cocktail using vivo-PMO chemistry was employed to correct the SCCC 521 $\mathrm{T}$ reading frame in myogenic cells. RT-PCR analysis showed treatment with vivo-PMOs targeting exons 4, 5, 6, and 7 generated the Mini-Gamma reading frame-corrected transcript (red arrowhead) with minimal residual expression of the mutant 521 $\mathrm{T}$ transcript (black arrowhead). Arrows in schematic denote the location of the RT-PCR primers, and the expected amplicon lengths are indicated. (B) Normal control myogenic cells were treated with the same multiexon-skipping vivo-PMO cocktail. RT-PCR analysis showed expression of the Mini-Gamma transcript (red arrowhead), indicating that normal SCCG transcript could be skipped using vivo-PMOs. Expression of the SGCG transcript in untreated cells is indicated (black arrowhead). (C and D) Sequence analysis of PCR products confirmed the generation of the Mini-Gamma transcripts in each of the mutant cell lines, with the splicing of SGCG exons 3 and 8 demonstrating the removal of exons $4,5,6$, and 7 from the mature transcripts.

internally truncated Mini-Gamma transcript (Figure 7B). IFM imaging detected Mini-Gamma protein expression in reprogrammed LGMD 2C mutant myotubes 3 days after treatment with vivo-PMO (Figure 7C). Protein expression was observed throughout the myotube, including at the plasma membrane (Supplemental Figure 7, A and B). Image analysis confirmed Mini-Gamma expression relative to reprogrammed cells transfected with nontargeting control vivo-PMOs (Figure 7D). The reprogrammed LGMD 2C patient cells were treated for 3 days with either reading frame-correcting vivo-PMOs or nontargeting control vivo-PMOs, then subjected to a hypo-osmotic shock to induce creatine kinase (CK) release. Treatment with vivo-PMOs to restore the reading frame resulted in a decrease in CK release in response to the hypo-osmotic shock, indicative of increased membrane integrity after the restoration of $S G C G$ expression (Figure 7E). We tested reprogrammed 521 $\Delta \mathrm{T}$, ex5/6del, and ex6del fibroblasts, using 
A
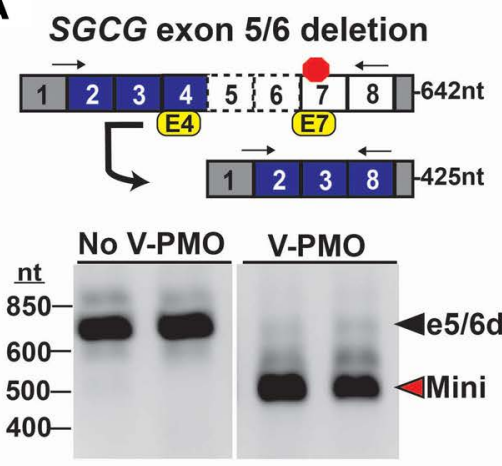

D

SGCG exon $5 / 6$ deletion

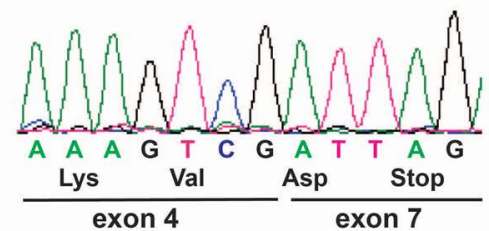

\section{Exon 5/6 deletion Mini-Gamma}

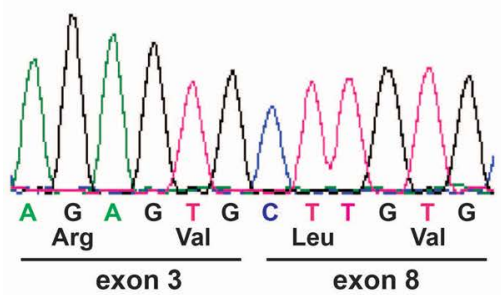

B
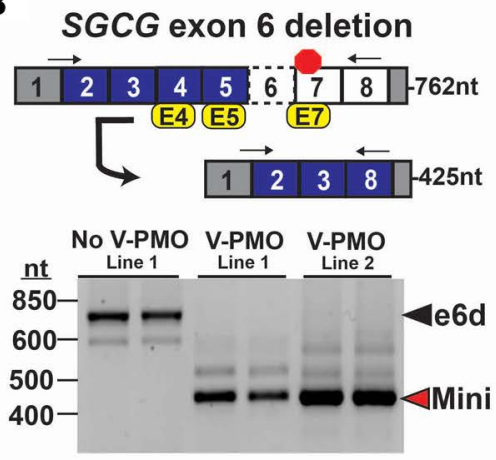

E

SGCG exon 6 deletion

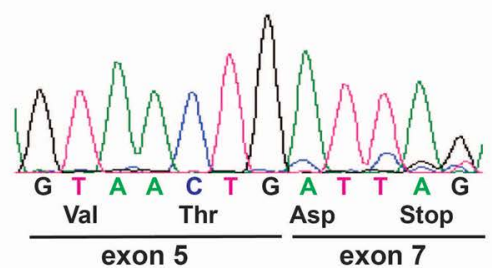

Exon 6 deletion Mini-Gamma

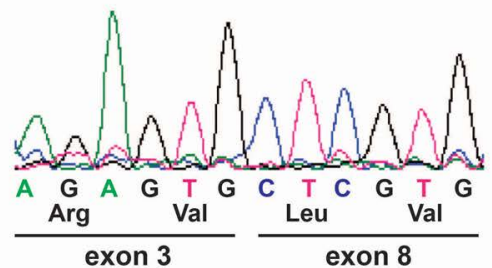

C
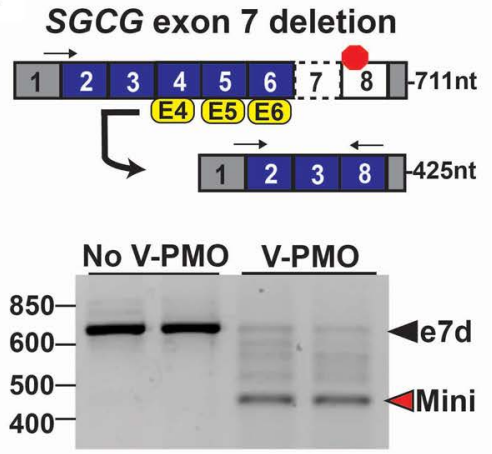

$\mathbf{F}$

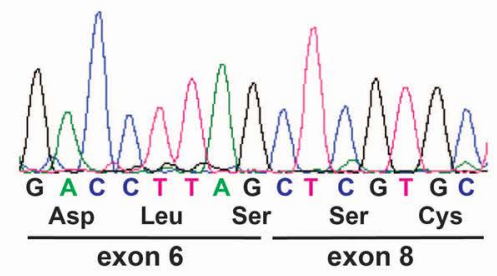

Exon 7 deletion Mini-Gamma

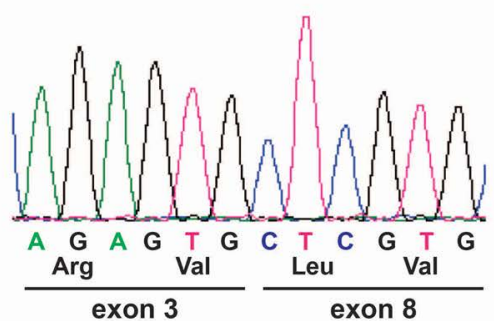

Figure 5. Reading frame correction of multiple SCCG frameshift mutations. (A-C) Top: Mutation-specific multiexon-skipping strategies were designed to correct the SGCG reading frame of multiple unrelated LGMD 2C patients whose primary SGCG mutations include (A) the deletion of exons 5 and 6; (B) the deletion of exon 6; and (C) the deletion of exon 7. Myogenic cells were treated with vivo-PMO exon skipping cocktails. Arrows denote the location of the RT-PCR primers, and the expected amplicon lengths are indicated. (A-C) Bottom: RT-PCR analysis confirmed the mutant SCCC transcripts were expressed in the reprogrammed cells at the expected size, consistent with their exon deletion status (black arrowheads). Cells treated with the mutation-specific vivo-PMOs cocktails demonstrated predominate expression of the reading frame-corrected Mini-Gamma transcript (red arrowheads). (D-F) Sequence analysis of RT-PCR products: (D) a deletion of exons 5 and 6; (E) a deletion of exon 6; and (F) a deletion of exon 7. Sequence analysis also confirmed the generation of the Mini-Gamma transcript in each of the mutant cell lines, with the splicing of SGCG exons 3 and 8 demonstrating the removal of (D) exons 4 and 7; (E) exon 4, 5, and 7; and (F) exons 4, 5 and 6 from the mature transcripts.

this same $\mathrm{CK}$ release assay. Treatment with reading frame-correcting vivo-PMOs resulted in a nonstatistically significant trend toward decreased CK release (Supplemental Figure 8, A-C). The trend toward increased CK release likely reflects that only a subset of the myotubes in culture expressed Mini-Gamma protein. However, taken together, these data demonstrate that exon skipping-mediated reading frame correction of human SGCG is feasible in human cell culture and protective against stress (Figure 7F).

\section{Discussion}

Exon skipping for $L G M D 2 C$. Exon splice-modulating therapy has arrived in the clinic with the recent approval of AONs for the treatment of DMD and SMA $(24,39)$. The field of RNA-targeting therapeutics continues to expand with new splice-switching oligonucleotide therapies designed to treat multiple disorders (40). In the current study, we evaluated an AON-mediated exon-skipping strategy to treat LGMD 2C. This approach required the bypass of SGCG exons 4, 5, 6, and 7 to generate an internally 
A

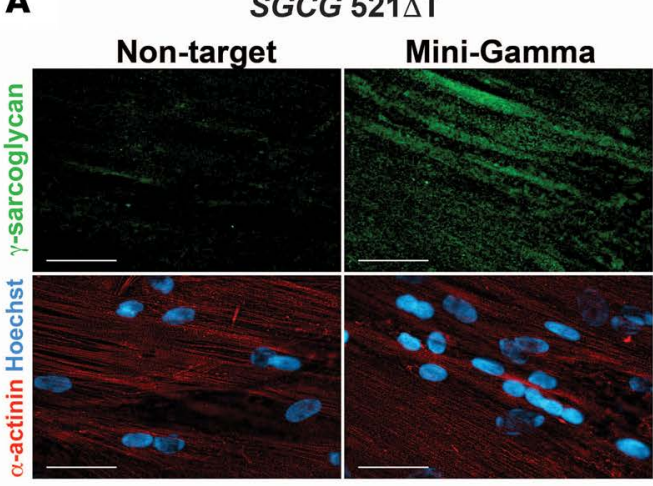

C

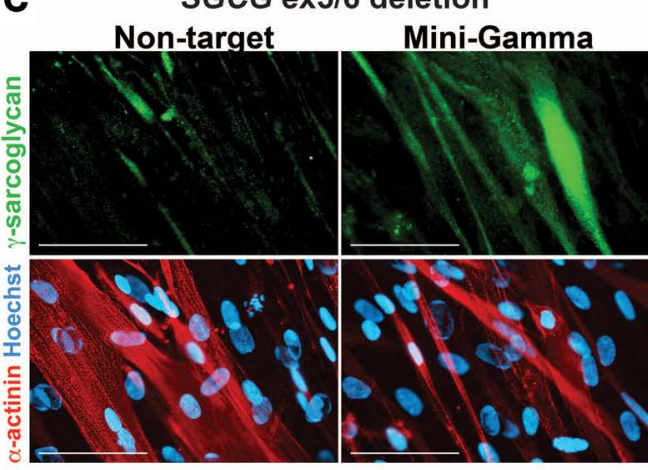

E

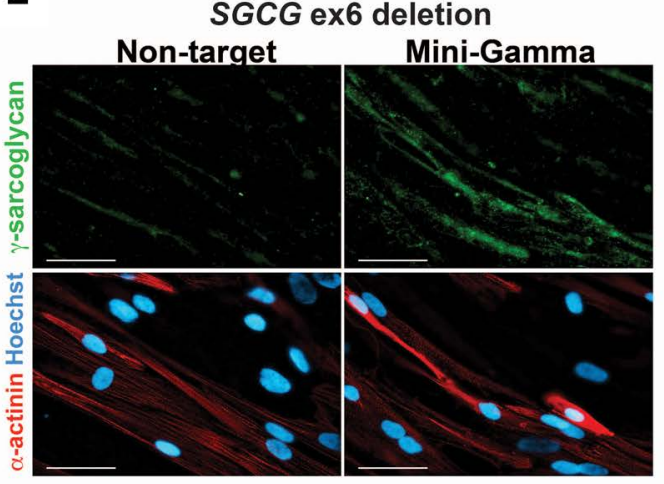

B

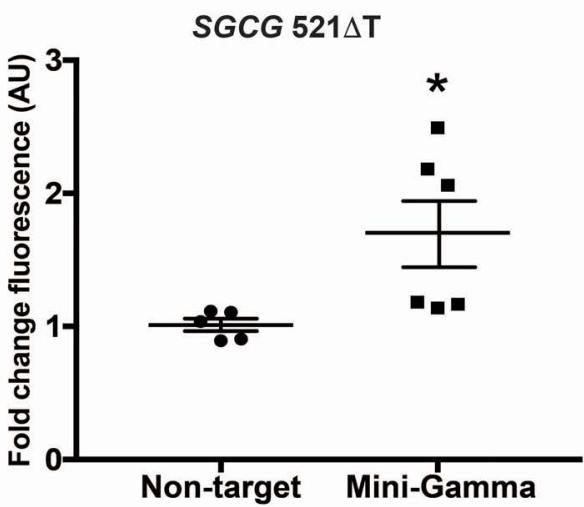

D

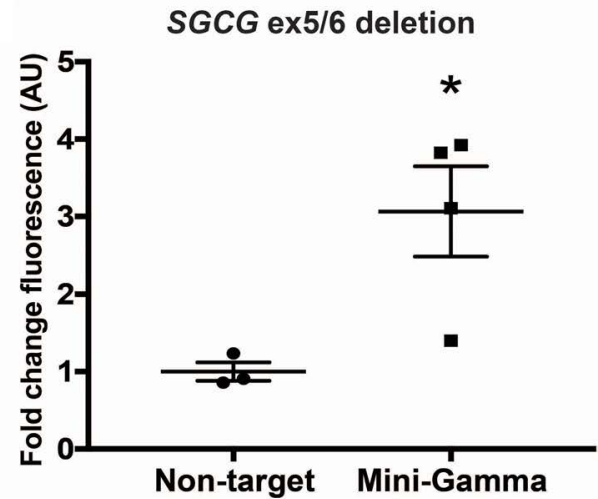

$\mathbf{F}$

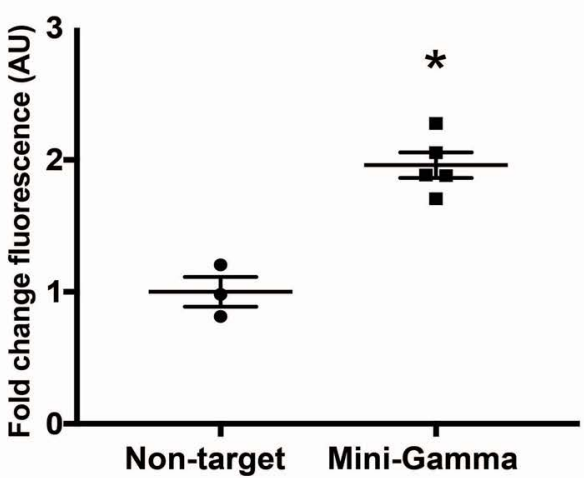

Figure 6. Restoration of SGCG expression as Mini-Gamma protein. Reprogrammed fibroblasts were treated for 3 days with either a nontargeting control vivo-PMO (nontarget) or a mutation-specific vivo-PMO exon skipping cocktail (Mini-Gamma). (A) Representative IFM images of reprogrammed $521 \Delta \mathrm{T}$ cells that were treated as indicated. Treatment with reading frame-correcting vivo-PMOs resulted in the expression of the Mini-Gamma protein (green). Myotubes were labeled with $\alpha$-actinin (red), and nuclei were labeled with Hoechst 3342 (blue). (B) Image analysis demonstrated a significant increase in Mini-Gamma fluorescence after treatment with vivo-PMOs that corrected the transcript reading frame ( $n$ = 6) as compared with nontargeting controls $(n=5)$. (C and $\mathbf{D})$ Reprogrammed cells harboring a deletion of SGCG exons 5 and 6 (ex5/6del) were treated as indicated. IFM image analysis demonstrated a significant increase in Mini-Gamma fluorescence after treatment with vivo-PMOs that corrected the reading transcript reading frame $(n=4)$ as compared with nontargeting controls $(n=3)$. (E and F) Reprogrammed cells harboring a deletion of SGCG exons 6 (ex6del) were treated as indicated. IFM image analysis demonstrated a significant increase in Mini-Gamma fluorescence after treatment with vivo-PMOs that corrected the transcript reading frame $(n=5)$ as compared with nontargeting controls $(n=3)$. Data represent the mean $\gamma$-sarcoglycan fluorescence per $\alpha$-actinin-positive area normalized to the mean of the untreated group. A minimum of 3 independent fields were analyzed for each sample. ${ }^{*} P<0.05$ as determined by 2 -tailed Student's $t$ test. Data represent the mean \pm SEM. Scale bars: $50 \mu M$. 
A

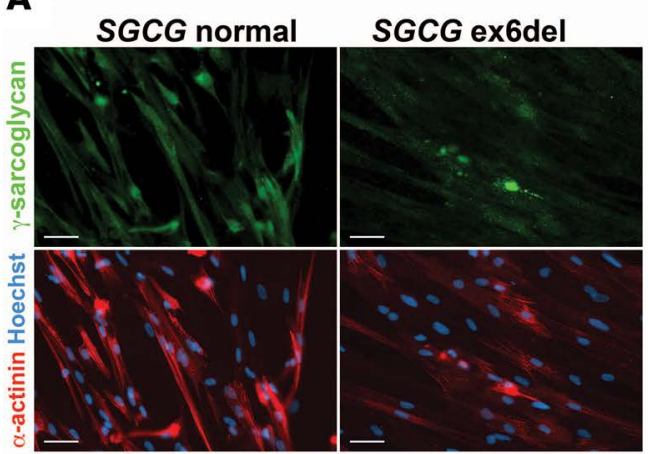

C

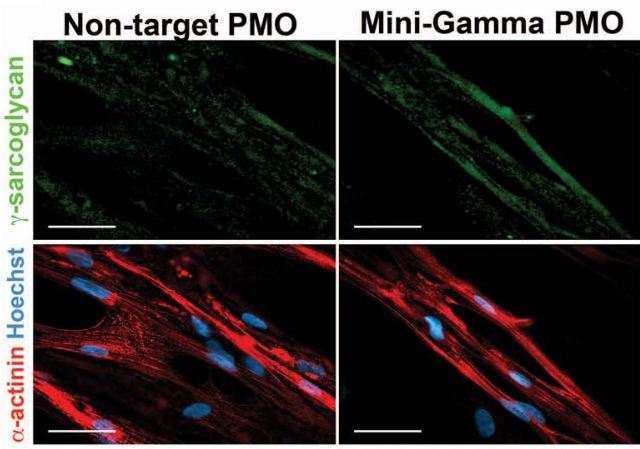

E

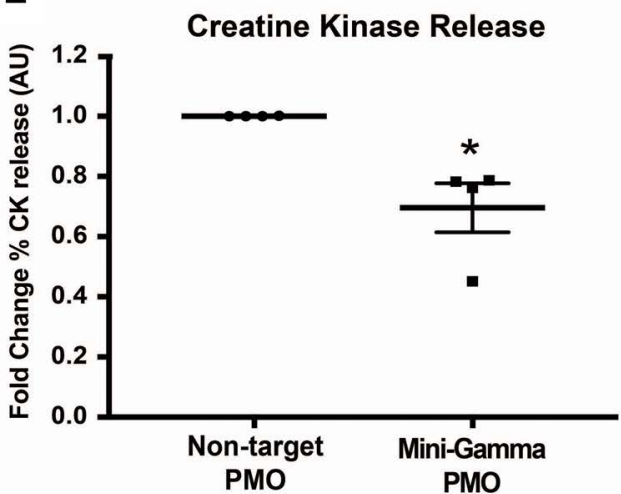

B

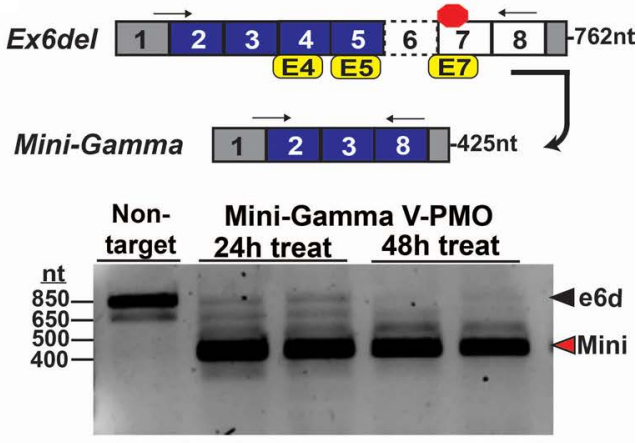

D

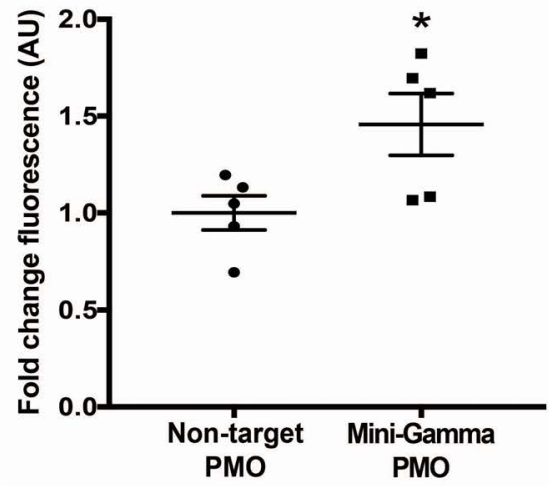

$\mathbf{F}$

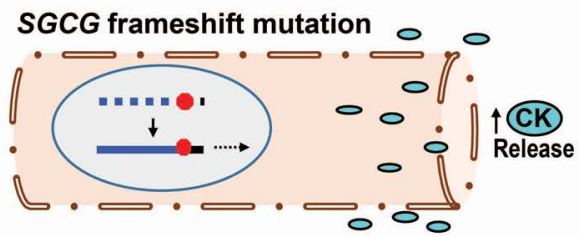

Readframe correction

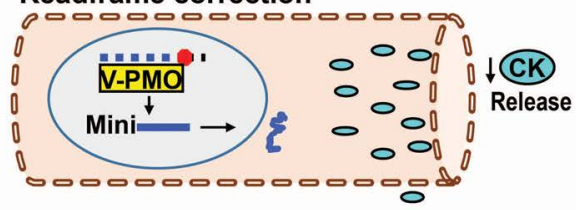

Figure 7. SGCG reading frame correction in urine-derived cells (UDCs). UDCs from a normal control subject and an LGMD 2 C patient with a deletion of exon 6 (ex6del) were reprogrammed into a myogenic lineage. (A) $\gamma$-Sarcoglycan protein (green) was detected by IFM in the reprogrammed normal control myotubes but not in reprogrammed ex6del cells. $\alpha$-Actinin, red; nuclei, blue. (B) RT-PCR analysis demonstrated reading frame-corrected Mini-Camma transcript expression (red arrowhead). (C) Representative IFM images showed the restoration of $\gamma$-sarcoglycan protein expression in cells treated with Mini-Gamma vivo-PMOs. (D) Significant increase in $\gamma$-sarcoglycan protein fluorescence was observed after treatment with vivo-PMOs $(n=5)$ as compared with nontargeting control vivo-PMOs $(n=5)$. A minimum of 3 independent fields were analyzed for each sample. (E) To assess membrane stability in response to vivo-PMO treatment, reprogrammed cells were challenged with hypo-osmotic shock and membrane leak was monitored by release of creatine kinase (CK). Vivo-PMO treatment significantly decreased the relative amount of CK release consistent with increased membrane stability. Data represent the percent of CK released relative to the total CK from 4 independent experiments ( $n=3-4$, for each). Data are presented as the mean CK released in cells treated with exon-skipping vivo-PMOs relative to the mean in cells treated with a nontargeting vivo-PMO. (F) Model depicting the increased membrane stability that resulted from vivo-PMO-mediated reading frame correction of an SGCG frameshift mutation. ${ }^{*} P<0.05$ as determined by 2 -tailed Student's $t$ test. Data represent the mean \pm SEM. Scale bars: $50 \mu \mathrm{M}$.

truncated product. A multiexon-skipping cocktail of vivo-PMO oligomers successfully generated the reading frame-corrected Mini-Gamma transcript in myogenically reprogrammed cells derived from a patient with the most common $S G C G 521 \Delta \mathrm{T}$ mutation. Notably, this strategy is applicable to any mutation occurring in $S G C G$ exons 4, 5, 6, and 7, and this approach was used to correct the reading frame of multiple distinct $S G C G$ frameshift mutations. Successful correction of the reading frame resulted in the 
restoration of SGCG protein expression as Mini-Gamma. These results demonstrate that exon skipping is a viable therapeutic approach for the treatment of LGMD 2C caused by mutations occurring in SGCG exons 4-7, which encompasses the majority of known mutations.

An exon-skipping strategy must generate a functional internally truncated protein. We previously used transgenic expression of the internally truncated murine Mini-Gamma to demonstrate partial correction of many aspects of muscular dystrophy in a mouse model of LGMD 2C (30). In these studies, Mini-Gamma maintained proper interaction with components of the DGC, improved membrane integrity, and rescued the dystrophic phenotype in both fly and mouse models of the disease (30). Mini-Gamma retains the complete cytosolic and transmembrane domains. Importantly, Mini-Gamma retains the cysteine-rich C-terminal extracellular domain, which is known to play a role in sarcoglycan complex formation and plasma membrane targeting $(9,41-43)$. The high homology between the human and mouse SGCG genes supported that this strategy should work on human SGCG. AON-mediated expression of Mini-Gamma in human $S G C G$ cells led to a reduction in the amount of CK released in response to hypo-osmotic shock, indicating a functional rescue of muscle membrane stability.

Vivo-PMOs appeared to have superior skipping compared with 2OMePS AONs. Chemically modified AONs are used to promote compound stability and avoid RNase digestion. In this study, we observed minimal multi-exon skipping using $20 \mathrm{MePS}$ AONs. This result was attributed primarily to the negligible skipping of exon 6 that was observed in both normal control and SGCG 521 $\triangle$ T cells. SGCG exon 6 is only 73 nucleotides in length, and by $\mathrm{m}$-fold is predicted to adopt a mainly closed secondary structure at the mRNA level (44). The sequence characteristics limited the number of potential AON candidates that could successfully target the exon. Altering the $2 O \mathrm{MePS} A O N$ length or target sequence could result in improved single and multi-exon skipping of exon 6.

Several studies have suggested that PMOs have better exon-skipping efficacy compared with 2OMePS in the targeting of specific dystrophin exons, and the difference is thought to be sequence dependent (4547). Unmodified PMOs were not assessed in the current study due to their difficulty of uptake in cultured cells (48). Instead, only vivo-PMOs were tested to enhance cell delivery. Improved molecular correction was observed using vivo-PMOs targeting the same exon sequences as the 2OMePS AONs. Single vivoPMO treatment showed robust skipping of exon 6 in both the $521 \Delta \mathrm{T}$ and normal control cells, and the multiexon-skipping cocktail targeting exons 4, 5, 6, and 7 generated predominate expression of the Mini-Gamma transcript with minimal expression of intermediate products.

The results also suggest that exon 7 has a high propensity toward skipping, even in the absence of AONs. Single vivo-PMO treatments targeting exons 4, 5, and 6 resulted in double-exon-skipping events, which each included exon 7 . Splice-site analysis did not indicate any anomalies in the splice acceptor site for exon 7; however, there are 2 regions, one intronic and one exonic, that had canonical splice repressor motifs without an adjacent enhancer motif (49). Thus, there may be competition between the exon 7 and exon 8 splice acceptor sites. This could reduce the number and/or concentration of AONs needed for therapeutic effect, since only a low concentration of exon 7-directed sequences would be needed.

Challenges for in vivo translation. Although multi-exon skipping was possible in a myogenic cell model, the delivery of multiple AONs in vivo is expected to face several hurdles. First, the previously published $\mathrm{Sgcg}$ mouse model is not molecularly suitable for testing exon skipping, as it was engineered to lack exon 2 (50). While studies in a canine and mouse models of DMD demonstrated the ability to skip multiple exons of the dystrophin gene using multi-AON cocktails $(51,52)$, sufficient dosing and potential side effects could limit the human translation of these observations. Advancements in oligonucleotide chemistries and strategies have sought to improve the efficacy and delivery of AON delivery. For example, the tri-cyclo AON modification demonstrated superior exon skipping efficiency as compared with 2OMePS AONs and PMOs in a mouse model of DMD, including access to the heart and brain (53). AONs have also been conjugated to ligands, nanoparticles, cell penetrating peptides, or covalently modified delivery moieties such as the octa-guanidine dendrimers (54). While toxicity concerns need to be addressed, these modifications represent continued progress toward the enhanced delivery of AONs at lower concentrations.

\section{Methods}

Cell culture. Primary fibroblasts were isolated from skin biopsies taken from 5 LGMD 2C patients. Primary fibroblasts from a healthy control subject were obtained from the American Type Culture Collection (ATCC, CCD-1127Sk). Primary UDCs were isolated from the urine of deidentified healthy volunteers and an LGMD 2C patient harboring a deletion of SGCG exon 6, as previously described (38). Primary 
fibroblasts were cultured in proliferation media consisting of DMEM (Thermo Fisher Scientific, 11955065) supplemented with 15\% FBS (Thermo Fisher Scientific, 26140-079). Primary UDCs were cultured in UDC proliferation media as described (38).

Lentiviral constructs and transduction. Primary fibroblasts were cotransduced with an hTert lentivirus containing a puromycin selection cassette (Applied Biological Materials [ABM], G200) and a iMyoD lentivirus as previously described (30). The $521 \Delta \mathrm{T}$ fibroblast cell line was transduced with the single iMyoD lentivirus (MOI 200). The iMyoD lentiviral construct was provided by Jeffrey Chamberlain (University of Washington, Seattle, Washington, USA), and packaged by the Northwestern Skin Disease Research Center (Chicago, Illinois, USA).

Myogenic reprogramming of human fibroblasts. Myogenic reprogramming of fibroblasts was performed as previously described with minor modifications (30). Briefly, iMyoD-transduced cells were seeded onto 24-well culture plates (Thermo Fisher Scientific, 353047) or coverslips (Thermo Fisher Scientific, 12-54581 ) at a density of 30,000 cells $/ \mathrm{cm}^{2}$ in proliferation media. When cells reached confluence, the media was replaced with proliferation media supplemented with $5 \mu \mathrm{M} 4 \mathrm{OH}$-tamoxifen (MilliporeSigma, H7904). After 48 hours, the media was replaced with differentiation media consisting of DMEM supplemented with 2.5\% Normal Horse Serum (NHS, Thermo Fisher Scientific, 161050-122), 1\% insulin-transferrin-selenium (MilliporeSigma, 41400-045), and $1 \mu \mathrm{M}$ tamoxifen. After 3 days, the media was replaced with differentiation media without tamoxifen, and it was exchanged every 3 days.

IFM. Prior to imaging, culture plates or coverslips were washed twice with DPBS (Thermo Fisher Scientific, 14190-144), fixed in ice cold methanol for 2 minutes, rinsed with DPBS, and blocked for 1 hour at $4^{\circ} \mathrm{C}$ in DPBS supplemented with $10 \%$ NHS and $0.1 \%$ triton (MilliporeSigma, X-100). Primary antibody incubations were performed in blocking buffer (DPBS supplemented with $5 \%$ NHS and $0.1 \%$ triton) at room temperature for 2 hours or overnight at $4^{\circ} \mathrm{C}$. Secondary antibody incubations were performed in blocking buffer at room temperature for 1 hour. Nuclei were counterstained with Hoechast 3342 (Thermo Fisher Scientific, H3570) diluted 1:10,000 in DPBS. Coverslips were mounted with Vectashield mounting media (Vector Laboratories, H-1000). Imaging was performed using the Zeiss Axio Observer Z1 microscope using the $20 \times$ objective, or the Zeiss Axio Observer A1 microscope, using $40 \times$ or $63 \times$ objectives with ZEN 2 software (version 2011; Zeiss). Images were processed using FIJI (NIH) and Adobe Photoshop CC.

IFM antibodies. Primary antibodies were as follows: rabbit polyclonal anti-MyoD (1:1,000, Santa Cruz Biotechnology Inc., sc-304), mouse monoclonal anti-desmin (1:1,000, MilliporeSigma, D1033), mouse monoclonal anti- $\alpha$-actinin (1:1,000, MilliporeSigma, A7811), mouse monoclonal anti- $\beta$-sarcoglycan (1:250, Leica Biosystems, B-SARC-L-CE,clone BETASARC1/5B1), mouse monoclonal anti- $\delta$-sarcoglycan $(1: 250$, Leica Biosystems, D-SARC-CE, clone DELTASARC/12C1), and rabbit polyclonal anti- $\gamma$-sarcoglycan $(1: 1,000)(8)$. Secondary antibodies included Alexa Fluor 488 donkey anti-mouse (A21202), Alexa Fluor 594 donkey anti-mouse (A21203), Alexa Fluor 488 donkey anti-rabbit (A21206), and Alexa Fluor 594 donkey anti-rabbit (A21207) (all 1:1,000, Thermo Fisher Scientific).

2OMePS design and transfection. 2OMePS AON were synthesized as 25-mers by Integrated DNA Technologies (IDT) following sequence guidelines outlined in ref. 36. AON sequences and their exon targets are listed in Table 1. The $20 \mathrm{MePS}$ AONs were transfected into fibroblasts in serum free media using Lipofectamine 3000 (Thermo Fisher Scientific) at a ratio of $2 \mu \mathrm{l}: 1 \mu \mathrm{g}$ DNA. After 6 hours, the serum free media was replaced with differentiation media. For reading frame correction, 2OMePS AONs targeting exons 4, 5, 6, and 7 were cotransfected at the dosages listed in Supplemental Table 1. Single 2OMePS AONs or multiexon-skipping AON cocktails were transfected into fibroblasts on differentiation day 9. Cells were isolated for analysis after 36-48 hours.

Vivo-PMO design and transfection. PMOs were designed according to described guidelines and synthesized as 25-mer vivo-PMOs by GeneTools. Specific AON sequences and their exon targets are listed in Table 1. A nontargeting vivo-PMO standard control was purchased from GeneTools (CCTCTTACCTCAGTTACAATTTATA). Vivo-PMO were resuspended in ultrapure water to a stock concentration of 0.5 $\mathrm{mM}$. For cell culture application, vivo-PMO were diluted in serum free media and then directly added to cells cultured in differentiation media. For reading frame correction, vivo-PMOs were cotransfected as indicated in Supplemental Table 1. Single vivo-PMOs or multiexon-skipping AON cocktails were transfected into fibroblasts on differentiation day 9 or 10. Cells were isolated for analysis as indicated.

RNA analysis. Prior to isolation, cells were washed once with DPBS. Total RNA was isolated in $1 \mathrm{ml}$ of TRIzol (Thermo Fisher Scientific, 15596018) according to the manufacturer's instructions and included the 
addition of glycogen $(10 \mu \mathrm{g} / \mathrm{ml})$ to the isopropanol before the RNA precipitation. The RNA was reverse-transcribed using qSCRIPT cDNA Supermix (Quanta Biosciences, 95048-025). To detect SGCG and Mini-Gamma, Taqman based reverse transcription PCR (RT-PCR) was used to amplify $50 \mathrm{ng}$ of complementary DNA (cDNA) with the following primer set ex1/2Fwd 5'-TCTAAGATGGTGCGTGAGCAG-3' and ex8R 5'-GCCACAGACAGGTACAGCTT-3'. PCR was performed by 35 cycles of $95^{\circ} \mathrm{C}\left(40\right.$ seconds), $57^{\circ} \mathrm{C}(30$ seconds), and $72^{\circ} \mathrm{C}$ ( 60 seconds). The PCR products were separated on a $1.5 \%$ agarose gel (Denville Scientific Inc., CA3510-8) supplemented with $0.5 \mu \mathrm{g} / \mathrm{ml}$ ethidium bromide. Gels were imaged with either the UVP transluminator (BioSpectrum) or the FluorChem E (Protein Simple).

Sequence analysis. RT-PCR was performed using the SGCG primer set as described. The PCR products were separated on a 1\% low-melt agarose gel (Lonza, 50080). Individual PCR bands were excised from the gel and extracted using the Qiaquick Gel Extraction Kit (Qiagen, 28706). Sanger sequencing of the purified products was conducted by ACGT Inc. The sequences were analyzed using Lasergen 13 software from DNASTAR. Some isolated products were reamplified prior to sequencing. For these samples, 5-10 ng of the purified product was reamplified as described above. For this PCR, the number of cycles was reduced to 25 and the annealing temperature was increased by $3^{\circ} \mathrm{C}$.

Splice-site analysis. Human and murine $\gamma$-sarcoglycan genes were analyzed for exon splicing elements encoded in exons 7 and 8, along with $100 \mathrm{bp}$ of the intronic region upstream of each exon. Splice-site analysis was conducted using the Human Splicing Finder software (www.umd.be/HSF3/) and the Ensembl transcript database $(49,55)$.

Mini-Gamma Image Analysis. IFM was performed as described above. Imaging was performed using a Zeiss Axio Observer A1 microscope, using 40× or 63× objectives with ZEN 2 software (version 2011; Zeiss). Images were processed using FIJI (NIH) and Adobe Photoshop CC. Quantitation of fluorescence was performed from multiple fields per condition using FIJI (NIH) and is presented as mean $\gamma$-sarcoglycan fluorescence per $\alpha$-actinin-positive area normalized to the mean of the nontargeted group.

Immunoblotting. iMyoD-transduced cells were reprogrammed in 6-well culture plates and treated with vivo-PMOs as indicated. Total protein was isolated in radioimmunoprecipitation assay (RIPA) buffer (Thermo Fisher Scientific, 89900) supplemented with a protease inhibitor cocktail (Roche Diagnostics, 11836170001). Briefly, culture media was removed, and cells were washed twice with ice-cold PBS. RIPA buffer was added to the cells, and they were collected using a cell scraper (Thermo Fisher Scientific, 08-100-241). Cell isolates were then sonicated using the Bioruptor 300 (Diagenode). Three 15 -second pulses were performed at the medium setting. Cells were then centrifuged at $4^{\circ} \mathrm{C}$ for 10 minutes at $15,000 \mathrm{~g}$, and the supernatant was collected. Protein samples were denatured using $4 \times \mathrm{NuP}$ AGE LDS sample buffer supplemented with reducing agent (Thermo Fisher Scientific, NP0007 and NP0004). Proteins were resolved on precast 4-12\% Bis-Tris gels (Thermo Fisher Scientific, NP0336), and then transferred to Immu-Blot PVDF membranes (Bio-Rad, 1620177). Membranes were blocked for 1 hour at room temperature with Starting Block T20 blocking buffer (Thermo Fisher Scientific, 37543), followed by incubation with primary antibody diluted in T20 blocking buffer for either 2 hours at room temperature or overnight at $4^{\circ} \mathrm{C}$. After primary incubation, the membranes were washed 3 times, for 20 minutes each, at room temperature with TBS containing $0.1 \%$ Tween-20. The membranes were then incubated for 1 hour with a goat anti-mouse or goat anti-rabbit HRP secondary antibodies diluted in T20 blocking buffer, followed by 3 washes. Enhanced chemiluminescence was performed with the SuperSignal West Pico Plus (Thermo Fisher Scientific, 34580) and imaged using the FluorChem E (Protein Simple) imaging system. Primary antibodies were as follows: rabbit polyclonal anti- $\gamma$-sarcoglycan $(1: 1,000)$ (8); rabbit polyclonal anti- $\gamma$-sarcoglycan $(1: 1,000$, Abnova; MaxPab H00006445-D01); and mouse monoclonal anti- $\gamma$-tubulin (1:1,000, MilliporeSigma, T5326/GTU-88). Secondary antibodies included HRP conjugated goat anti-mouse and goat anti-rabbit (Jackson ImmunoResearch; 115-035-003 and 111-035-144).

Mini-Gamma overexpression. HEK293T cells were obtained from the ATCC (CCD-1127Sk) and cultured in DMEM supplemented with 10\% FBS and $1 \times$ Pen/Strep, described above. Human Mini-Gamma was synthesized and cloned into the pcDNA3.1(+) vector by GenScript. Human Mini-Gamma was transfected into HEK293T cultured in Opti-MEM (Thermo Fisher Scientific, 31985062) using Lipofectamine 3000 according to the manufacturer's instructions (Thermo Fisher Scientific, L3000015). Cells were cultured in the presence of transfection media and reagents for 6 hours; then, the media was replaced with fresh growth media. Total protein was isolated as described 3 days after transfection. 
Reprogrammed UDCs. Primary UDCs were transduced with the $\mathrm{iMyoD}$ lentivirus as previously described (38). Myogenic reprogramming of iMyoD-transduced urine cells was performed as previously described with minor modifications. Briefly, culture plates and coverslips were coated with $8 \mu \mathrm{g} /$ $\mathrm{cm}^{2}$ and $24 \mu \mathrm{g} / \mathrm{cm}^{2}$ collagen I (MilliporeSigma, C3867), respectively. Cells were seeded at a density of 50,000 cells $/ \mathrm{cm}^{2}$ in proliferation media and cultured until confluent. On the day of induction, cells were overlaid with Growth Factor Reduced Matrigel (Corning, 354230) and exposed to $2.5 \mu \mathrm{M}$ tamoxifen in proliferation media. After 48 hours, the media was replaced with Hydrocortisone dexamethasone (HD) differentiation media supplemented with $1 \mu \mathrm{M}$ tamoxifen. Half of the media was replenished every 3 days with HD differentiation media without tamoxifen. After 14 days, media was replaced with differentiation media (DMEM; $2.5 \%$ NHS; $1 \%$ insulin-transferrin-selenium), and half of the media was replenished every 3 days.

Vivo-PMO treatment of UDCs. Vivo-PMO were diluted in serum free media and then directly added to urine cells cultured in differentiation media. For reading frame correction, vivo-PMOs were cotransfected as indicated in Supplemental Table 1. Multiexon-skipping AON cocktails or nontargeting control vivoPMO were transfected into UDCs on differentiation day 25. Cells were isolated for analysis as indicated. RNA analysis, IFM, and image analysis were performed as described above.

Hypo-osmotic shock and CK release. Reprogrammed fibroblasts and UDCs were challenged with hypo-osmotic shock through the replacement of culture media with deionized water $(56,57)$. Cells were cultured in 24-well plates and treated with vivo-PMO as indicated. Prior to treatment (3-12 hours), cells were washed with DPBS and differentiation media was replaced $(500 \mu 1)$. To induce hypo-osmotic shock, $350 \mu 1$ of media was removed and replaced with $350 \mu$ of deionized water. Cells were exposed to hypo-osmotic conditions for $20-30$ minutes at $37^{\circ} \mathrm{C}$. The supernatant, containing the released $\mathrm{CK}$ fraction, was collected and placed on ice. Following supernatant collection, deionized water was added to the remaining cell fraction and submitted to 3 cycles of freeze-thaw to rupture the cell membranes and release the remaining CK into solution. Prior to assay, each fraction was subject to centrifugation at $1,000 \mathrm{~g}$ for 10 minutes at $4^{\circ} \mathrm{C}$. CK was measured using a kinetic assay, used according to manufacturers' instructions (BioAssay System, ECPK-100). The cellular CK fraction and the released CK fraction represent the total CK. Released CK represented the percent of the $\mathrm{CK}$ in the supernatant fraction relative to the total $\mathrm{CK}$.

Statistics. Prism (GraphPad Software) was used for data analysis. A 2-tailed Student's $t$ test was used to compare results between 2 groups. $P<0.05$ was considered significant.

Study approval. Written informed consent was obtained from all human subjects. All work was approved by and conducted under the IRBs of the University of Chicago, Northwestern University, and the Biosciences Institute Ethics Committee on Human Subject at Universidade de São Paulo, which serve as the ethics boards for each institution. All studies were conducted in compliance with the Helsinki Declaration.

\section{Author contributions}

EJW performed the in vitro experiments. ARD analyzed the IFM data. EYK contributed to the isolation and culture of human cells. AHV contributed to the in silico analysis. LMDC contributed to the acquisition of patient samples and patient information. MZ, MV, and RCMP contributed to the acquisition, isolation, and culture of human cells. ARD, MJP, and QQG made critical contributions to the manuscript preparation. EMM directed the study. EJW and EMM designed the study, analyzed the data, and wrote the manuscript.

\section{Acknowledgments}

We thank the Kurt+Peter Foundation for facilitating these studies and the patients and families for participation. We thank Jeff Chamberlain for providing the inducible MyoD construct. We thank Jon Moulton, of GeneTools, for help in oligonucleotide design. This work was supported by NIH R01HL61322, NIH T32 HD009007, FAPESP-CEPID, and CNPq.

Address correspondence to: Eugene J. Wyatt or Elizabeth M. McNally, Center for Genetic Medicine, Northwestern University Feinberg School of Medicine, 303 E. Superior Street, Chicago, Illinois 60611, USA. Phone: 312.503.5600; Email: eugene.wyatt@northwestern.edu (EJW) or elizabeth.mcnally@northwestern.edu (EMM). 
1. Fayssoil A, et al. Natural History of Cardiac and Respiratory Involvement, Prognosis and Predictive Factors for Long-Term Survival in Adult Patients with Limb Girdle Muscular Dystrophies Type 2C and 2D. PLoS ONE. 2016;11(4):e0153095.

2. Kirschner J, Lochmüller H. Sarcoglycanopathies. Handb Clin Neurol. 2011;101:41-46.

3. Noguchi S, et al. Mutations in the dystrophin-associated protein gamma-sarcoglycan in chromosome 13 muscular dystrophy. Science. 1995;270(5237):819-822.

4. Ervasti JM, Campbell KP. Membrane organization of the dystrophin-glycoprotein complex. Cell. 1991;66(6):1121-1131.

5. Ozawa E, Mizuno Y, Hagiwara Y, Sasaoka T, Yoshida M. Molecular and cell biology of the sarcoglycan complex. Muscle Nerve. 2005;32(5):563-576.

6. Cohn RD, Campbell KP. Molecular basis of muscular dystrophies. Muscle Nerve. 2000;23(10):1456-1471.

7. Ervasti JM, Campbell KP. A role for the dystrophin-glycoprotein complex as a transmembrane linker between laminin and actin. J Cell Biol. 1993;122(4):809-823.

8. McNally EM, et al. Mild and severe muscular dystrophy caused by a single gamma-sarcoglycan mutation. Am J Hum Genet. 1996;59(5):1040-1047.

9. McNally EM, et al. Mutations that disrupt the carboxyl-terminus of gamma-sarcoglycan cause muscular dystrophy. Hum Mol Genet. 1996;5(11):1841-1847.

10. Straub V, Bushby K. Therapeutic possibilities in the autosomal recessive limb-girdle muscular dystrophies. Neurotherapeutics. 2008;5(4):619-626.

11. Hoffman EP, Brown RH, Kunkel LM. Dystrophin: the protein product of the Duchenne muscular dystrophy locus. Cell. 1987;51(6):919-928.

12. Koenig M, et al. The molecular basis for Duchenne versus Becker muscular dystrophy: correlation of severity with type of deletion. Am J Hum Genet. 1989;45(4):498-506.

13. Monaco AP, Bertelson CJ, Liechti-Gallati S, Moser H, Kunkel LM. An explanation for the phenotypic differences between patients bearing partial deletions of the DMD locus. Genomics. 1988;2(1):90-95.

14. Fanin M, Danieli GA, Vitiello L, Senter L, Angelini C. Prevalence of dystrophin-positive fibers in 85 Duchenne muscular dystrophy patients. Neuromuscul Disord. 1992;2(1):41-45.

15. Hoffman EP, Morgan JE, Watkins SC, Partridge TA. Somatic reversion/suppression of the mouse mdx phenotype in vivo. $J$ Neurol Sci. 1990;99(1):9-25.

16. Nicholson LV. The "rescue" of dystrophin synthesis in boys with Duchenne muscular dystrophy. Neuromuscul Disord. 1993;3(56):525-531.

17. Kole R, Krainer AR, Altman S. RNA therapeutics: beyond RNA interference and antisense oligonucleotides. Nat Rev Drug Discov. 2012;11(2):125-140.

18. Chan JH, Lim S, Wong WS. Antisense oligonucleotides: from design to therapeutic application. Clin Exp Pharmacol Physiol. 2006;33(5-6):533-540.

19. Cirak S, et al. Exon skipping and dystrophin restoration in patients with Duchenne muscular dystrophy after systemic phosphorodiamidate morpholino oligomer treatment: an open-label, phase 2, dose-escalation study. Lancet. 2011;378(9791):595-605.

20. Goemans NM, et al. Systemic administration of PRO051 in Duchenne's muscular dystrophy. N Engl J Med. 2011;364(16):1513-1522.

21. Mendell JR, et al. Eteplirsen for the treatment of Duchenne muscular dystrophy. Ann Neurol. 2013;74(5):637-647.

22. Voit T, et al. Safety and efficacy of drisapersen for the treatment of Duchenne muscular dystrophy (DEMAND II): an exploratory, randomised, placebo-controlled phase 2 study. Lancet Neurol. 2014;13(10):987-996.

23. Aartsma-Rus A, Krieg AM. FDA Approves Eteplirsen for Duchenne Muscular Dystrophy: The Next Chapter in the Eteplirsen Saga. Nucleic Acid Ther. 2017;27(1):1-3.

24. Aartsma-Rus A. FDA Approval of Nusinersen for Spinal Muscular Atrophy Makes 2016 the Year of Splice Modulating Oligonucleotides. Nucleic Acid Ther. 2017;27(2):67-69.

25. Clayton NP, et al. Antisense Oligonucleotide-mediated Suppression of Muscle Glycogen Synthase 1 Synthesis as an Approach for Substrate Reduction Therapy of Pompe Disease. Mol Ther Nucleic Acids. 2014;3:e206.

26. Gedicke-Hornung C, et al. Rescue of cardiomyopathy through U7snRNA-mediated exon skipping in Mybpc3-targeted knock-in mice. EMBO Mol Med. 2013;5(7):1128-1145.

27. Gramlich $M$, et al. Antisense-mediated exon skipping: a therapeutic strategy for titin-based dilated cardiomyopathy. $E M B O M o l$ Med. 2015;7(5):562-576.

28. Igreja S, Clarke LA, Botelho HM, Marques L, Amaral MD. Correction of a Cystic Fibrosis Splicing Mutation by Antisense Oligonucleotides. Hum Mutat. 2016;37(2):209-215.

29. Lee JM, et al. Modulation of LMNA splicing as a strategy to treat prelamin A diseases. J Clin Invest. 2016;126(4):1592-1602.

30. Gao QQ, et al. Reengineering a transmembrane protein to treat muscular dystrophy using exon skipping. J Clin Invest. 2015;125(11):4186-4195.

31. Morcos PA, Li Y, Jiang S. Vivo-Morpholinos: a non-peptide transporter delivers Morpholinos into a wide array of mouse tissues. BioTechniques. 2008;45(6):613-618.

32. Kimura E, et al. Cell-lineage regulated myogenesis for dystrophin replacement: a novel therapeutic approach for treatment of muscular dystrophy. Hum Mol Genet. 2008;17(16):2507-2517.

33. Dunckley MG, Manoharan M, Villiet P, Eperon IC, Dickson G. Modification of splicing in the dystrophin gene in cultured Mdx muscle cells by antisense oligoribonucleotides. Hum Mol Genet. 1998;7(7):1083-1090.

34. van Deutekom JC, et al. Antisense-induced exon skipping restores dystrophin expression in DMD patient derived muscle cells Hum Mol Genet. 2001;10(15):1547-1554.

35. Wilton SD, et al. Specific removal of the nonsense mutation from the mdx dystrophin mRNA using antisense oligonucleotides. Neuromuscul Disord. 1999;9(5):330-338.

36. Aartsma-Rus A. Overview on AON design. Methods Mol Biol. 2012;867:117-129.

37. Douglas AG, Wood MJ. Splicing therapy for neuromuscular disease. Mol Cell Neurosci. 2013;56:169-185.

38. Kim EY, Page P, Dellefave-Castillo LM, McNally EM, Wyatt EJ. Direct reprogramming of urine-derived cells with inducible MyoD for modeling human muscle disease. Skelet Muscle. 2016;6:32. 
39. McNally EM, Wyatt EJ. Mutation-Based Therapy for Duchenne Muscular Dystrophy: Antisense Treatment Arrives in the Clinic. Circulation. 2017;136(11):979-981.

40. Havens MA, Hastings ML. Splice-switching antisense oligonucleotides as therapeutic drugs. Nucleic Acids Res. 2016;44(14):6549-6563.

41. Chan YM, Bönnemann CG, Lidov HG, Kunkel LM. Molecular organization of sarcoglycan complex in mouse myotubes in culture. J Cell Biol. 1998;143(7):2033-2044.

42. Chen J, et al. Identification of functional domains in sarcoglycans essential for their interaction and plasma membrane targeting. Exp Cell Res. 2006;312(9):1610-1625.

43. Shi W, Chen Z, Schottenfeld J, Stahl RC, Kunkel LM, Chan YM. Specific assembly pathway of sarcoglycans is dependent on beta- and delta-sarcoglycan. Muscle Nerve. 2004;29(3):409-419.

44. Zuker M. Mfold web server for nucleic acid folding and hybridization prediction. Nucleic Acids Res. 2003;31(13):3406-3415.

45. Fletcher S, Honeyman K, Fall AM, Harding PL, Johnsen RD, Wilton SD. Dystrophin expression in the mdx mouse after localised and systemic administration of a morpholino antisense oligonucleotide. J Gene Med. 2006;8(2):207-216.

46. Heemskerk HA, et al. In vivo comparison of 2'-O-methyl phosphorothioate and morpholino antisense oligonucleotides for Duchenne muscular dystrophy exon skipping. J Gene Med. 2009;11(3):257-266.

47. McClorey G, et al. Induced dystrophin exon skipping in human muscle explants. Neuromuscul Disord. 2006;16(9-10):583-590.

48. Amantana A, Iversen PL. Pharmacokinetics and biodistribution of phosphorodiamidate morpholino antisense oligomers. Curr Opin Pharmacol. 2005;5(5):550-555.

49. Desmet FO, Hamroun D, Lalande M, Collod-Béroud G, Claustres M, Béroud C. Human Splicing Finder: an online bioinformatics tool to predict splicing signals. Nucleic Acids Res. 2009;37(9):e67.

50. Hack AA, et al. Gamma-sarcoglycan deficiency leads to muscle membrane defects and apoptosis independent of dystrophin. J Cell Biol. 1998;142(5):1279-1287.

51. Aoki Y, et al. Bodywide skipping of exons 45-55 in dystrophic mdx52 mice by systemic antisense delivery. Proc Natl Acad Sci USA. 2012;109(34):13763-13768.

52. Yokota T, et al. Efficacy of systemic morpholino exon-skipping in Duchenne dystrophy dogs. Ann Neurol. 2009;65(6):667-676

53. Goyenvalle A, et al. Functional correction in mouse models of muscular dystrophy using exon-skipping tricyclo-DNA oligomers. Nat Med. 2015;21(3):270-275.

54. Sardone V, Zhou H, Muntoni F, Ferlini A, Falzarano MS. Antisense Oligonucleotide-Based Therapy for Neuromuscular Disease. Molecules. 2017;22(4):E563.

55. Flicek P, et al. Ensemb1 2013. Nucleic Acids Res. 2013;41(Database issue):D48-D55

56. Sinha B, et al. Cells respond to mechanical stress by rapid disassembly of caveolae. Cell. 2011;144(3):402-413.

57. Wang B, et al. Membrane blebbing as an assessment of functional rescue of dysferlin-deficient human myotubes via nonsense suppression. J Appl Physiol. 2010;109(3):901-905. 